$12-1988$

\title{
Integrating Multiple Criteria Decision-Making Models Into the Decision Support System Framework for Marketing Decisions
}

\author{
Rajshekhar G. Javalgi \\ Cleveland State University, r.javalgi@csuohio.edu \\ Hemant K. Jain \\ University of Wisconsin - Milwaukee
}

Follow this and additional works at: https://engagedscholarship.csuohio.edu/busmarkt_facpub

Part of the Marketing Commons, and the Organizational Behavior and Theory Commons How does access to this work benefit you? Let us know!

Publisher's Statement

(c) 1988 Wiley-Blackwell

\section{Original Citation}

Javalgi, R. G., \& Jain, H. K. (1988). Integrating multiple criteria decision making models into the decision support system framework for marketing decisions. Naval Research Logistics, 35, 6, 575-596.

\section{Repository Citation}

Javalgi, Rajshekhar G. and Jain, Hemant K., "Integrating Multiple Criteria Decision-Making Models Into the Decision Support System Framework for Marketing Decisions" (1988). Marketing. 50.

https://engagedscholarship.csuohio.edu/busmarkt_facpub/50

This Article is brought to you for free and open access by the Browse Business Faculty Books and Publications by Topic at EngagedScholarship@CSU. It has been accepted for inclusion in Marketing by an authorized administrator of EngagedScholarship@CSU. For more information, please contact library.es@csuohio.edu. 


\title{
INTEGRATING MULTIPLE CRITERIA DECISION-MAKING MODELS INTO THE DECISION SUPPORT SYSTEM FRAMEWORK FOR MARKETING DECISIONS
}

\author{
Rajshekhar G. Javalgi, Cleveland State University \\ H. K. Jain
}

This article was originally published in:

Javalgi, R. G., \& Jain, H. K. (1988). Integrating multiple criteria decision making models into the decision support system framework for marketing decisions. Naval Research Logistics, 35, 6, 575596.

Post-print standardized by MSL Academic Endeavors, the imprint of the Michael Schwartz Library at Cleveland State University, 2012

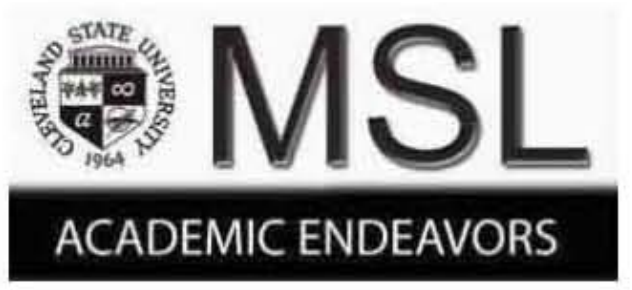




\title{
Integrating Multiple Criteria Decision Making Models into the Decision Support System Framework for Marketing Decisions
}

\author{
Rajshekhar G. Javalgi \\ College of Business Administration, Cleveland State University, \\ Cleveland, Ohio 44115 \\ Hemant K. Jain \\ School of Business Administration, University of Wisconsin-Milwaukee, \\ Milwaukee, Wisconsin 53201
}

\begin{abstract}
A large number of multiple criteria decision making (MCDM) models have evolved over the past decade. This field now seems to have reached a stage of maturity. However, the managerial community has not yet extensively adopted these models in solving practical decision problems. The present article focuses on integrating the MCDM models within the decision support system (DSS) framework to encourage greater use of these models. A DSS framework and the criteria used for the choice of a model is discussed. Based on these criteria MCDM models generally used in the marketing field are evaluated. The possibility of using a mixture of MCDM models within the DSS framework is also explored. Following this, the role of the MCDM models in DSS is delineated. It is argued that, within the problem-solving process, the confluence of MCDM models and DSS plays a vital role in developing high-quality solutions.
\end{abstract}

\section{INTRODUCTION}

In the context of problem solving which encompasses structured and semistructured problems, a decision support system (DSS) promises to be a significant advancement $[5,51]$. The effectiveness and success of DSS is accentuated in the following ways: (i) in DSS, attempts have been made to create convenient human interfaces; (ii) the user is given control of the problem-solving process; and (iii) algorithms of various decision-making models are being incorporated into the system to aid effective decision making. Keen and Morton [26, pp. 57-58] describe DSS as "the key point, for a DSS is to support or enhance the manager's decision making ability." Thus, a DSS attempts to combine the use of models or analytic techniques with traditional data access and retrieval functions. The systems focus specifically on features that make them easy to use by people unfamiliar with computers in an interactive mode. The essential features of a DSS are flexibility and usability [26].

The role of the models in DSS are to support the discovery of an adoptable plan of action, rather than to dictate what the decisions should be $[2,5]$. Very often the recommendations generated by the model will be relevant only to a limited part of a 


\section{Integrating Multiple Criteria Decision Making Models}

much larger problem. They may be subject to numerous modifications that are required by the consideration of other factors that could not be explicitly incorporated into the model. Thus a DSS should support interaction between the user and the modeling component of the system, which allows the examination of intermediate results and accommodates subjective judgement during the problem-solving process.

As a result of theoretical developments and practical applications, multiple criteria decision making (MCDM) models are becoming more and more popular in the management decision-making paradigm $[9,56,60]$. The present study focuses on integrating MCDM models within the DSS framework to encourage greater use of these models.

Multiple-criteria decision problems are prevalent in many business fields, including marketing, operations management, finance, accounting, etc. The reality of the management decision-making environment tends to be complex, ill-structured, difficult to formulate, and potentially the most rewarding. Because of this a model of any reasonable richness will return to multiple criteria, forcing the management (or decision maker) to incorporate a variety of criteria in evaluating alternatives.

On the plane of theoretical developments, the field of MCDM seems to be in a mature stage. However, the managerial community has not completely acknowledged its importance to decision-making processes which can benefit from the use of models enriched with multiple objectives. Some of the reasons for the lack of practical applications of these models may be

(i) the underlying assumptions used to derive these models might not comply with the reality in which most managers make decisions

(ii) these models are generally made available through academic literature, which requires knowledge of sophisticated mathematical techniques to utilize them in the business field

(iii) an efficient and easy to use mechanism for providing the required human input during the problem-solving process is not available.

A consensus to integrate MCDM models and DSS has been acknowledged. Zeleny [56, p. 472], for example, notes that a "DSS-MCDM marriage is already upon us, the two participants provide a glimpse at decision making in the eighties and beyond." The confluence of MCDM-DSS creates a system that the decision makers can use for solving ill-structured problems with conflicting objectives. To further strengthen the surge of interest in building an effective DSS, the utilization of MCDM models should be encouraged. Continuing this line of thought and following Zeleny's ideas, the present article attempts to address the following issues related to the development of MCDM-DSS's.

1. What criteria can be employed to evaluate the suitability of the use of a model in the DSS environment?

2. Can a single MCDM model or a combination of MCDM models (mixed models) be integrated within the DSS framework, and to what extent can these models respond to the characteristic of DSS?

3. What role do the MCDM models seek in DSS?

For this purpose, the MCDM models commonly used for marketing decisions have been reviewed and an evaluation of these models within the DSS framework is presented. The specific models discussed are state-of-the-art models generally used in marketing decisions.

A DSS framework and the criteria for the choice of a model is discussed in the next 


\section{Integrating Multiple Criteria Decision Making Models}

section. The difficulties in using traditional single-criterion models within the DSS framework are also discussed, followed by the problem-solving framework within DSS. Section 3 presents a review of the MCDM models, followed by a discussion of the possibility of using mixed models within DSS. In Section 4, the role of MCDM models in building decision-support systems is examined.

\section{THE DSS FRAMEWORK}

DSS's can be viewed as computer-based systems that lie at the intersection of two major evolutionary trends-data processing, which deals with managing data, and management science, which offers decision models. The confluence of these two trends enables a decision maker to devise high-quality solutions to what are often partially formulated (or ill-structured) problems.

Research in the area of DSS's often refers to the following reference criteria for judging the effectiveness and performance of the DSS [25, 26, 51].

1. A DSS should provide support for decision making, with emphasis on semistructured and unstructured decisions.

2. It should provide a variety of control to the decision maker, who should be able to control direction of solution and should be able to provide intermediate control information

3. It should assist in all phases of the decision-making process [50]; and it should support decisions which are interdependent as well as those that are independent.

4. It should be easy to use, which includes such characteristics as flexibility, user friendliness, requiring minimal user memory, error-tolerant, and nonthreatening.

The implications of the above list are profound when an isomorphism between the DSS and MCDM is considered. For the purpose of evaluating the use of MCDM models in the DSS framework, the criteria used for the choice of models in DSS is discussed here.

\section{Criteria for the Choice of a Model in DSS}

The modeling component of a DSS is the primary tool for supporting the design and choice phases of the problem-solving process. The activities generally supported by DSS are projection, deduction, analysis, creation of alternatives (suggestions), comparison of alternatives, optimization, and simulation. Some of these activities can best be supported by conceptual model building to be performed prior to the actual use of models. Landry, Pascot, and Briolat [31] define DSS problem as "a subjective representation conceived by a particular member of an organization when confronted with a reality which he perceives as unsatisfactory." They propose a design methodology which permits the operationalization of such a viewpoint. They also proposed a procedural framework of DSS which incorporates the whole problem-handling process, and shows how different types of modeling techniques may be useful at different stages in the process. In DSS the support for the problem-solving process depends on the feedback and interaction between the user and the modeling component. This should allow the examination of intermediate results, the accommodation of subjective judgement during the problem-solving process, and modification of the objective functions if the user's perception of the problem changes.

Various criteria have been proposed for the choice of a model. Dyer and Mulvey [11] propose an evaluation scheme based on five criteria. These are performance, realism/complexity, computational costs, information requirements, and ease of use. 
As a model becomes more approximate and sacrifices reality in order to gain information and computational advantages, in general the model must be run more times in order to gain insights. Thus a successful use of the model requires it to be flexible and interactive.

Barbosa and Hirko [2] specify some desirable features of a model used in DSS. They state that the structural changes in the problem occur as the user gains a better understanding of the problem variables and their interrelationships; this is because of the changing perception of the problem by the user. Thus the system should be able to accommodate the changing structure of the problem. Secondly, the system should be structured so that the user can utilize the subjective information during the solution generation phase. Another desirable feature of the system is to have rapid enough feedback (i.e., information flow back to the user) to enhance the problem-solving process. If a large amount of information or a large number of interactions are required at each step of the process, then this feedback to the user will be impaired. Barbosa and Hirko [2] grouped the capabilities required of the model for use in DSS into the following four categories:

(i) Control. The user should be given a spectrum of control. The control mechanism should allow the user to introduce subjective information as demanded by the problemsolution process.

(ii) Interface. The control parameters should be expressed in terms with which the user will be familiar. The user should have to think about only those parameters which relate directly to the problem-solving process.

(iii) Flexibility. The algorithmic and manual operations should be interchangeable in the sense that the user can develop part of a solution via manual methods and then continue with the algorithm or vice versa.

(iv) Feedback. The system should provide feedback essential for supporting human control of the process. The design process itself should make use of feedback.

\section{Difficulties in Using Traditional Models in DSS}

Traditionally models have been applied primarily to well-structured problems. In fact, one of the definitions of modeling activity is the conversion of ill-structured problems into well-structured problems. The view of traditional modeling can be depicted as

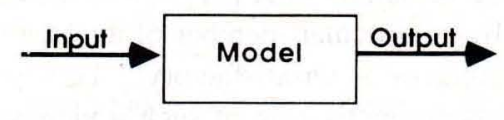

The structure of the model is the center of attention. Sprague and Carlson [51, p. 259] identified specific problems with model usage in DSS. Some of the relevant problems are

1. The necessary input data or parameters are often not available or are very difficult to generate.

2. The output from the model is often difficult to use. Even if it is in report form, it is usually static, hard to manipulate, and seldom in action-compelling form.

3. Generally, there is a minimum of interaction between the decision maker and 


\section{Integrating Multiple Criteria Decision Making Models}

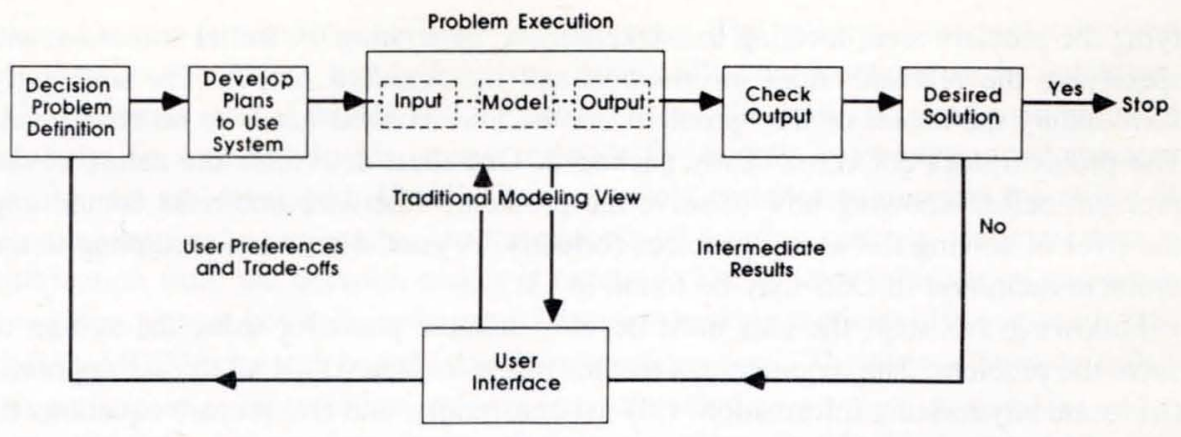

Figure 1. Decision problem solving using DSS.

the model. Even if the model is run in an interactive environment, the interaction is usually limited to supplying the necessary data or parameter values and selecting certain options for execution or output format. Thus, these modeling techniques assume that there is nothing to support, as they leave no discretion for the decision maker.

Also, considering a single objective function (profit, cost, utility, etc.), as in the classical mathematical optimization, presupposes that the decision maker's preferences and values are both complete and definite at the beginning of the decision process. Once the objective function is specified, there is no further demand on the decision maker's involvement. The decision maker can then say only yes or no to the final outcome. The solution of mathematical optimization is usually unique and no further effort on the part of the decision maker is required. Also these models do not allow consideration of parameters which cannot be represented explicitly in the model. Thus, these models are not really suitable for solving the type of semistructured problems which a decision support system aims at solving.

The portfolio of the decision maker's criteria often includes more than one objective or goal. For example, the process of deciding which automobile to purchase involves not only the actual choice among currently available makes, but, more importantly, the selection of the appropriate criteria of choice: price, mileage, projected maintenance costs, horsepower, appearance, expected resale value, overall appeal, etc. One of the approaches used to solve these multiple-criteria problems is to construct an all-encompassing objective function. This requires the assumption that decision makers' preferences and tradeoffs can be precisely measured and explicated. However, the decision maker's preferences and tradeoffs may develop in the course of the decision-making process and may depend on a given situation. This learning process is typically accompanied by consecutive modifications and specifications of one or more objectives. A properly designed DSS can easily support this kind of learning process.

\section{Problem Solving Framework within DSS}

Figure 1 represents a problem-solving framework within DSS. This is an extension of the traditional view of the model which incorporates additional steps required prior to input and after the output is generated. This also allows for intermediate input during the model execution. This permits the user interaction and flexibility required for solving most semistructured decision problems.

The problem-definition phase is both difficult and important. This involves identi- 
fying the problem area, locating the stakeholders, generating the initial scenarios, and specifying the relevant input information and the required output. The ambiguity surrounding the nature of the "problem" in the DSS context needs to be eliminated. The problem does not come neatly packaged. One must determine the nature of the problem before knowing how to solve the problem, otherwise one risks committing the error of solving the wrong problem correctly. A good discussion pertaining to the problem definition in DSS may be found in [31].

Following this step, the user must develop suitable plans for using the system to solve the problem. The requirements for this phase include (i) list all the assumptions, (ii) locate any missing information, (iii) list constraints, and (iv) prepare equations (if any). After this stage, the next step is to execute the model. During the model execution intermediate results are displayed to the user and the user can provide preferences and tradeoff information to help determine the search path. The next important step is to check the output. At this point, the user must ask the following questions: (i) are the magnitudes of the results reasonable? (ii) is it necessary to make changes or relax constraints? If the desired solution is achieved, the decision process ends; otherwise the user must make changes or redefine the problem.

Based on the above discussion of the desirable features of a model for DSS use, the following set of criteria to evaluate the currently used MCDM models for marketing decisions has been identified.

(1) Model Characteristics. Refers to how the values of attributes are considered and combined in the model. This also indicates how the problem parameters are generated.

(2) Availability of the Required Input. The models are evaluated based on the difficulty of generating the required input data.

(3) Nature of the Output. The output characteristics of the model refer to type and form of output provided by the model.

(4) User Control and Flexibility. Refers to the amount of control and flexibility provided to the user in selecting and changing model parameter values, obtaining intermediate results, and selecting alternative paths.

(5) Availability of packages. A software implementation of the model in a structured form helps significantly in the integration of the models in DSS.

\section{MULTIPLE CRITERIA DECISION MAKING MODELS}

The term multiple criteria is applied to decisions involving either multiple objectives or multiple criteria or both. These problems arise in many different decision situations; for example, in making a decision, one could be concerned about maximizing expected returns, minimizing total cost, or maximizing revenues, etc. In most cases, there are structural relationships among the objectives and perhaps some conflict among them. Also the tradeoffs between the objectives need to be considered. Another characteristic of these problems is that the objectives are apparently noncommensurable. MCDM models help the decision maker in this task. Mathematically, the MCDM problems can be represented as

$$
\begin{aligned}
& \max \left[f_{1}(x), f_{2}(x), \ldots, f_{k}(x)\right], \\
& \text { subject to } g_{i}(x) \leqq 0, i=1, \ldots, m ;
\end{aligned}
$$




\section{Integrating Multiple Criteria Decision Making Models}

where $x$ is an $n$-dimensional decision variable vector. The problem consists of $n$ decision variables, $m$ constraints, and $k$ objectives. Any or all of the functions can be nonlinear.

The ultimate goal of the MCDM methods is to find the most-preferred nondominated solution. To accomplish this, many methods for finding a complete nondominated solution set are developed. Usually it is extremely complex to measure the utility of all nondominated solutions, so, in the process of decision making, some preference information from the decision maker is required. The type of information and when it is given plays a key role in the actual decision making methods. Hwang et al. [18] classify MCDM research based upon these considerations. The ways a decision maker can participate in the solution process were classified as a priori, progressive and $a$ posteriori articulation of preference information from the decision maker. Among these, the methods based on progressive articulation of preference information, sometimes known as interactive programming, are particularly well suited for the semistructured nature of DSS problems. The important notions used in these models are nondominacy, proximity to ideal points, tradeoffs, satisfaction of goal achievements, and range of goal levels.

A nondominated solution means a feasible solution for which an increase in value of any one criterion can be achieved only at the expense of a decrease in value of at least one other criterion [56, p. 70]. A number of methods are proposed for generating the nondominated solution set; these are the weighing method, the $\varepsilon$-constraint method, and the multiobjective simplex method [56, Ch. 4]. These methods generate a set of nondominated solutions in steps so that the number of solutions the decision maker is required to compare at a given time is not very large. The decision makers can consider parameters not specified in the model, and use their own subjective judgment either to select a nondominated solution or to specify preference information for generating the next set of nondominated solutions. Thus an interaction with the decision maker is supported.

The ideal point of objective $i$ can be computed by maximizing $f_{i}(x)$, subject to $g_{i}(x) \leqq 0$ in (1). This is generally known as "anchor value," denoted by $x_{i}^{*}$. The collection of all such anchor values for $i=1, \ldots, n$ is known as the "ideal alternative" denoted as

$$
x^{*}=\left(x_{1}^{*}, \ldots, x_{n}^{*}\right) .
$$

The ideal alternative plays a prominent role in decision making. Although this is normally unattainable it provides a benchmark for comparison. If an ideal solution is found, it will be accepted with the highest level of confidence. The decision maker prefers the feasible solution closest to the perceived ideal. The typical methods which pursue the proximity to ideal points or some target points are the STEP method (STEM) [3], the sequential multiobjective problem-solving technique (SEMOPS) [36], the sequential information generator for multiple-objective problems (SIGMOP) [37], the method of displaced ideal solution [56, Chaps. 5 and 6], goal programming STEM [19], and interactive sequential goal programming (ISGP) [34].

When the explicit tradeoff information is given, the method proposed by Geoffrion [14] and interactive goal programming [3] can be used. These algorithms ask the decision maker to specify an overall preference function to resolve the conflict inherent in the given multiple criteria. The algorithm does not explicitly ask the whole preference function. Instead, local information about the preference function is requested to carry out the optimization. However, determination of the marginal rate of substitution 


\section{Integrating Multiple Criteria Decision Making Models}

(MRS) and step size can be quite difficult for the decision maker. Chankong and Haimes [8], and Zionts and Wallenius [61] try to generate the MRS rather than ask the decision maker to do so.

Some of the MCDM methods represent extensions of the single-criterion optimization technique (e.g., goal programming). Other types of multicriteria decision models are (i) methods based on the principles of decomposition and comparative judgments, and (ii) multiattribute choice models.

The decomposition principle calls for structuring the hierarchy to capture the basic elements of the problem. The analytic hierarchy process (AHP) is a multicriterion decision method for representing the basic elements of a problem in a hierarchic structure. This method calls for simple pairwise comparison judgments in order to develop priorities in each hierarchy. A discussion of this method follows.

\section{Analytic Hierarchy Process Model}

The analytic hierarchy modeling and measurement process recently developed by Saaty [43], is a method used to determine the relative importance of a set of criteria. The novel aspect of the analytic hierarchy process (AHP) is that it structures any complex multicriterion problem hierarchically.

When the hierarchy is designed to reflect likely choice-environment scenarios, objectives, and alternative product/market options, the AHP process can provide a multicriterion framework for marketers to better understand the decision process. Using a method for scaling [43], the relative importance weights of the elements in each level of the hierarchy, with respect to an element (e.g., criterion) of the next higher level, can be represented in a matrix of pairwise comparisons of the criteria. The entries of the matrix indicate the strength with which one element dominates another with respect to a given criterion. This scaling formulation is translated into a maximum eigenvalue problem. Solving this eigenvalue problem, a normalized and unique vector of weights for each level of the hierarchy, with respect to the criterion in the next level, is obtained. This may be transferred into a single composite vector of weights for the entire hierarchy. This vector measures the relative priority of all elements at the lowest level, which enables the accomplishment of the highest objective of the hierarchy. The relative priority weights can provide guidelines for the allocation of resources among the entities at the lower levels of the hierarchy.

AHP does not require judgements to be consistent. By consistency we mean the traditional requirement of transitivity of preferences (if Coke is preferred to Pepsi and Pepsi is preferred to 7-Up, then Coke must be preferred to 7-Up), but the actual intensity with which the preference is expressed transits through the sequence of objects in the comparison. For instance, if Coke is twice as preferable as Pepsi and Pepsi is three times as preferable as 7-Up, then Coke must be six times as preferable as 7-Up. This is what is referred to as cardinal consistency in the strength of preference. Inconsistency is a violation of proportionality, which may or may not entail violation of transitivity. AHP provides an index for measuring inconsistency for each matrix of comparisons and for the entire hierarchy. Knowledge of inconsistencies enables one to determine those judgments that may need reassessment. As a realistic representation of the situation in preference comparisons, one may wish to account for inconsistency in judgments because, despite people's best efforts, their feelings and preferences remain inconsistent and intransitive. 


\section{Integrating Multiple Criteria Decision Making Models}

When a group, often composed of people with different status levels, expertise and experience, uses AHP, the group may reach consensus on some issues after discussions about priorities. When they differ, their judgments can be combined after discussion by applying a geometric mean to these judgments. This is because the judgements themselves and their reciprocals must be viewed systematically [1]. The reciprocal of the geometric mean of a set of judgements is the geometric mean of the reciprocals. This is not true of the arithmetic mean. Saaty [43] points out that the use of the geometric mean is a less-desirable alternative; and several factors, including individual involvement and their knowledge about the problem area, may affect the results. To obtain the best results and/or improve consistency, Saaty [47] discusses some alternative methods. Other interesting issues, such as rank generation, preservation, and reversal in AHP are discussed in [46]. The AHP algorithm is available in a software product called "Expert Choice" (Decision Support Software, Inc., 1984). An evaluation of this model within the DSS framework, based on the criteria described in Section 2 , is presented in Table 1 .

Applications: The areas in which AHP is applied are diverse and numerous; they include marketing [54], portfolio selection [45], and health-care problems [44]. Another class of MCDM falls under the category of choice models.

\section{Multiattribute Choice Models}

Multiattribute choice models purport the integration of individual criteria across aspects (attributes) or alternative choices. A choice model is a procedure for selecting a product-alternative from a set consisting of a finite number of alternatives available to the decision maker in the marketplace. For instance, marketing decisions of selecting a computer or a car can be explained by a procedure that underlies the choice process. One can broadly classify choice models into two classes - the aspect- (attribute-) based models, which combine the attributes hierarchically or sequentially, and the multiattribute-utility-theory-(MAUT-) based techniques, which combine the attributes into one functional form through a specific utility formula.

Processing-by-Attribute (Aspect) Models. These models are based on the processing-by-attribute principle of the threshold concept. In this process, decision alternatives are evaluated on attribute or aspect one at a time, and unacceptable alternatives are eliminated hierarchically (sequentially) as they fail to meet the desired threshold on that aspect. The aspect-based processing models that are employed to solve marketing problems include the lexicographic, the elimination-by-aspect, and the maximum-likelihood hierarchical method. These methods, classified as sequential (hierarchical) elimination methods, are briefly expounded below.

Sequential Elimination Models. Work in such areas as psychology [53], information theory (Schroder et al. [48]), and artificial intelligence [39] posit that individuals often use sequential decision processes. The lexicographic, the elimination-by-aspects, the conjunctive, and the disjunctive models are developed to represent this process. These models are noncompensatory in the sense that a low value on one attribute will not be compensated by high values on other attributes.

In conjunctive and disjunctive models the decision maker sets up standards to be applied to the values on certain attributes. In a conjunctive approach which is characterized by and, all the standards must be acceptable in order for the alternative to

be acceptable. In disjunctive form, which is characterized by $o r$, the decision maker 


\section{Integrating Multiple Criteria Decision Making Models}

accepts any alternative which has one or more attribute values above a particular standard. The lexicographic approach also falls under the class of sequential elimination models. It is different from conjunctive and disjunctive approaches in that attribute values of the alternatives are directly compared at each decision point in the sequence. These sequential processing techniques are nonprobabilistic in structure and hence, choice outcome is not probabilistic. A well-known context-dependent probabilistic model in which the decision process is by attribute is the elimination-by-aspect (EBA) model proposed by Tversky [53].

The EBA Model. In this model each alternative is viewed as a collection of aspects that denote all valued attributes of the alternatives, including the quantitative attribute (e.g., price) and the nominal attribute (e.g., fish on the menu); the choice is described as a covert process of elimination [53]. The EBA characterizes the choice process as one of random sequential elimination of multiattribute alternatives until a single alternative remains. The selection process is a function of only those attributes that are not common to all the alternatives. The detailed discussion of this model can be found in Tversky [53].

There are some marketing applications of the lexicographic models (e.g., Russ [42], Bettman [4]); however, practical applications of the EBA model in marketing field are sparse. In the EBA model, the model builder must a priori know the critical values (cutoff values) of the aspects (attributes) for each individual. The question of obtaining this information and other data needed for practical implementation has yet to be addressed. However, the recent advancements in the field have offered new insights by presenting a methodology of estimating threshold values or cutpoints of the attributes. One such method known as the maximum-likelihood hierarchical model is discussed here.

Maximum Likelihood Hierarchical Model. Another class of noncompensatory lexicographic model, where the model builder does not have to know a priori the cutoff values of the attributes of the alternatives, is the maximum-likelihood hierarchical (MLH) model proposed by Gensch and Svestka [13]. The MLH model, like the EBA, is probabilistic and accommodates Tversky's observation that choice behavior is often inconsistent, hierarchical, and context dependent. MLH, which generates maximumlikelihood estimators of the aggregate cutoff values (threshold tolerances), operates in two distinct modes: (1) calibration (generating aggregate estimates of the threshold tolerances), and (2) prediction (employing the given estimates to predict individual responses). In the calibration mode, MLH generates the aggregate estimates of the threshold tolerances from information provided by a sample of individuals. In order to generate the calibrated coefficients, information obtained from each individual should include (i) rank order of the attributes, indicating the sequence in which they are considered, (ii) a set of self-perceived values of the given alternatives with respect to each attribute, and (iii) the individual's actual choice or final preference ranking of the alternatives. A discussion of the decision process, and how the MLH model generates aggregate estimates of the cutoff values of the criteria (attributes) is presented in the appendix.

The MLH Model is used to understand the underlying decision process of individuals and to predict their future choice distributions. While the EBA model in its present form is most suited for choice decisions in which key aspects (criteria) are dichotomous and unique to subsets of the alternatives, in the MLH model, the criteria are common 


\section{Integrating Multiple Criteria Decision Making Models}

to all choice alternatives. Though the MLH model is a useful counterpart of the EBA procedure, its use in the prediction model within a DSS framework requires high stability in the way individuals consider preferences. If such stability is not experienced, the MLH model may do little better than a simple additive multiattribute utility model in the prediction mode. The areas of application include transportation studies, industrial marketing studies [13], and consumer-behavior areas [12].

The sequential elimination methods presented above are built on the notion of noncompensatory behavior. An evaluation of these models in DSS framework is presented in Table 1. Another type of MCDM model is built on the multiattribute utility theory (MAUT) decision paradigm. The following discussion focuses on this topic.

\section{Multiattribute-Utility-Theory-Based Models}

Multiattribute utility theory (MAUT) is a well-known and widely accepted approach. MAUT analysis developed by Keeney and Raiffa [27] and others has shown great effectiveness in articulating value tradeoffs among criteria or attributes. In the analysis of marketing problems, the focus of marketing research is to understand and predict the behavior of consumers in the marketplace using the MAUT approach. The underlying assumptions and structure of the MAUT model allows practitioners and researchers to better understand the underpinnings of market behavior.

These models derive their mathematical function from a description space of choice outcomes, problem assumptions, and a decision maker's tastes onto a unidimensional scale of value, called utility [28]. The MAUT models can be linear or nonlinear. Let us define a set of alternatives as $A_{1}, A_{2}, \ldots, A_{j}$, with each one quantitatively described along $k$ criteria or attributes $Z_{1}, Z_{2}, \ldots, Z_{k}$, each of which has subjective importance $\beta_{i}$ to the decision maker. For computing the utility of an alternative $A_{j}$, the additive model takes the following form:

$$
U\left(A_{j}\right)=\beta_{0}+\sum_{i=1}^{k} \beta_{i} Z_{i}\left(A_{j}\right),
$$

where $Z_{i}\left(A_{j}\right)$ means alternative $j$ 's score or value of attribute $i$. The alternative that has the highest utility is preferred as the "rational" choice. The above additive form is widely used in practice because of its simplicity.

To discern the underlying market behavior of choosing from among discrete alternatives, commonly employed MAUT models are conjoint measurement [16], LINMAP [52], and Zionts method [59]. These MAUT methods are briefly discussed below.

LINMAP (Linear Programming Techniques for Multidimensional Analysis of Preferences). The LINMAP methodology, proposed by Srinivasan and Schocker [52], is used to analyze individual differences in preference judgments with regard to a set of stimuli (alternatives). In this model, the stimuli are represented as points in a space known as the multiattribute space. It assumes that the decision maker has an ideal point denoting a most-preferred location in the attribute space. Given two stimuli, an individual decision maker is presumed to prefer the stimulus (alternative) which is "closer" to his ideal point. As a measure of distance, either the Euclidean metric or the weighted Euclidean metric (where the dimensions are differently weighted for different individuals) is normally used. A linear-programming model is used to estimate the coordinates of his/her ideal point (in terms of the objective value) and the weights 


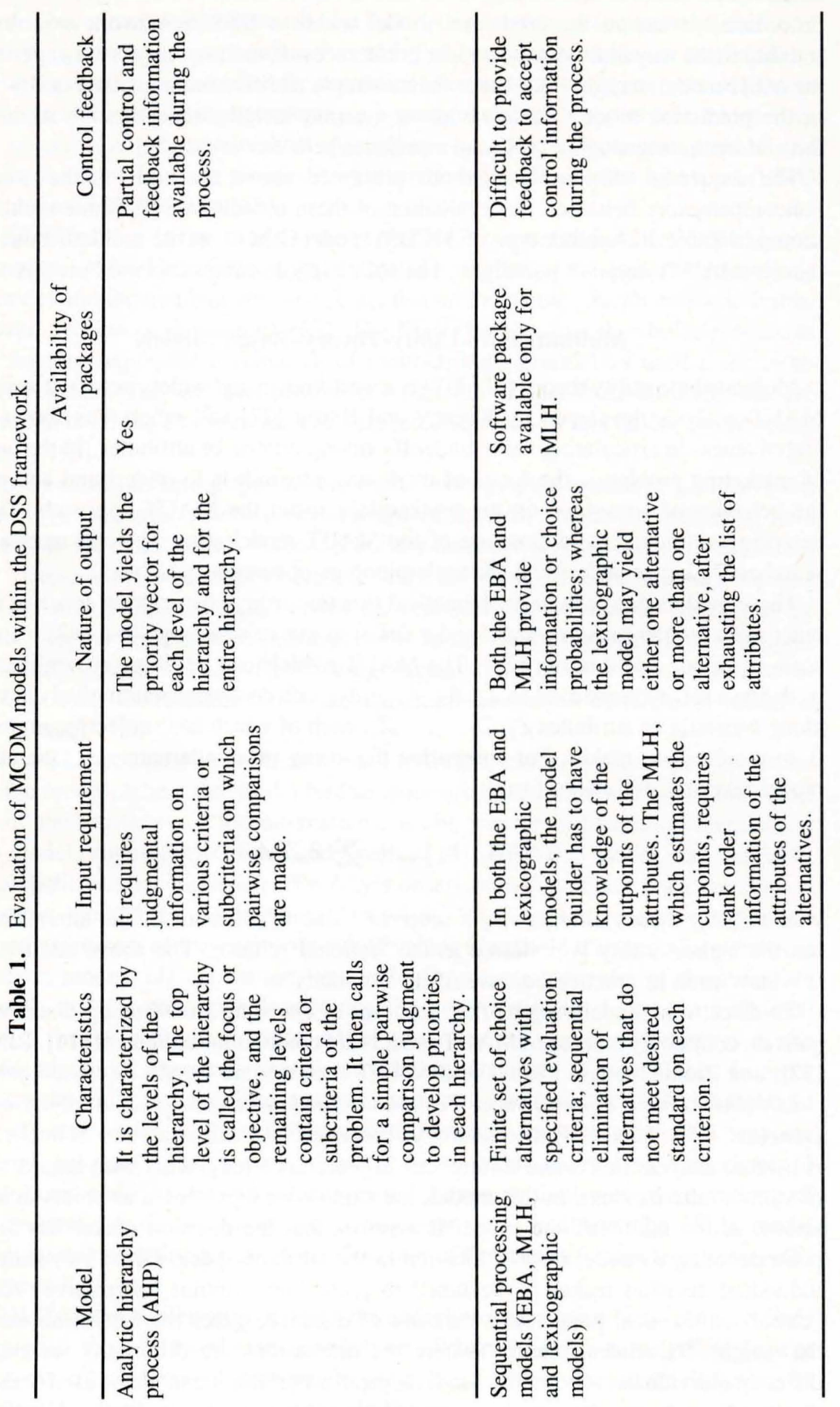




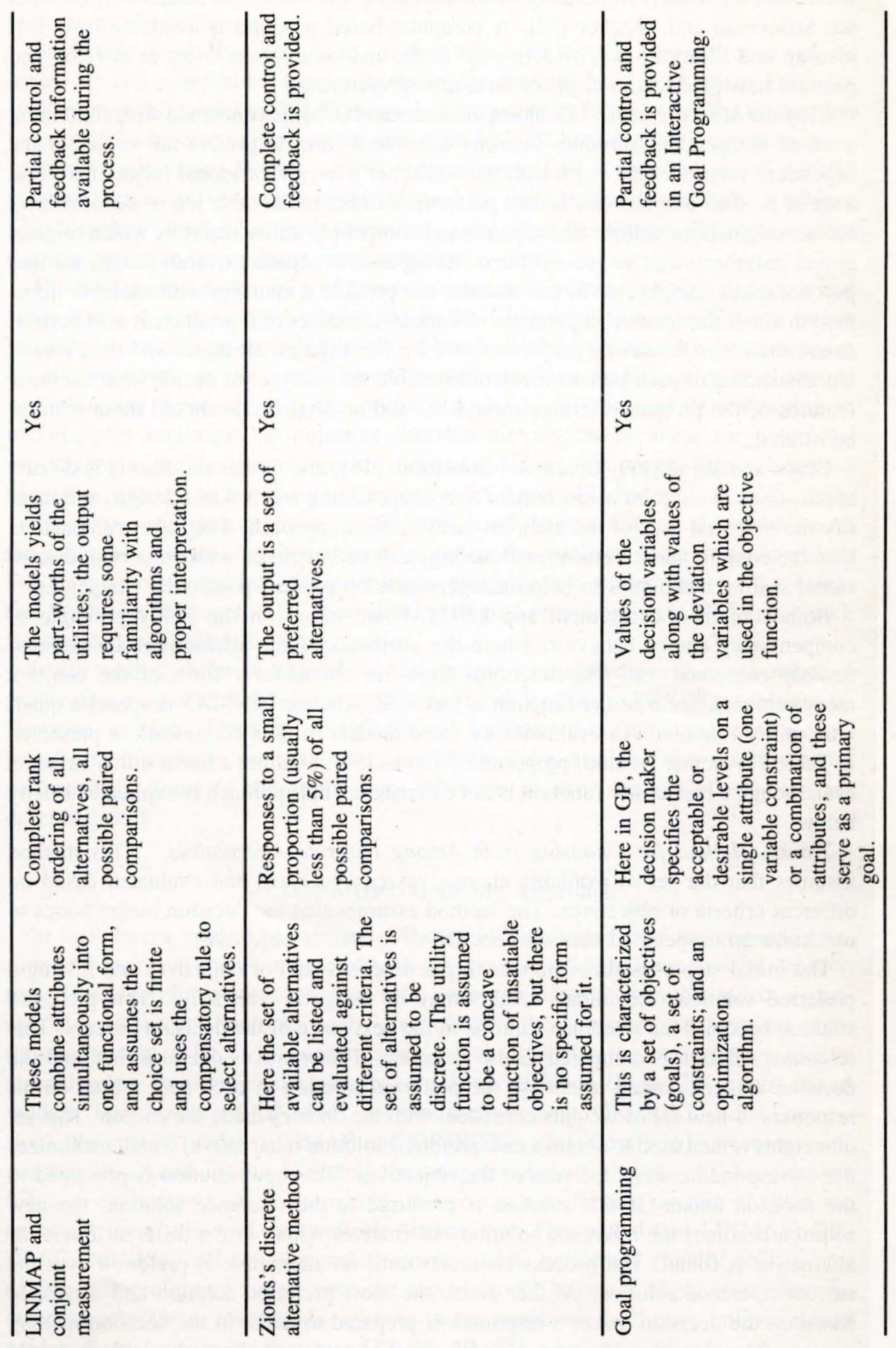




\section{Integrating Multiple Criteria Decision Making Models}

that reveal the relative importance of the attributes. For additional details of this model see Srinivasan and Shocker [52]. A computer-based program is available from Srinivasan and Shocker. It is widely used in the marketing area to try to develop and position new products in terms of consumer preferences.

Conjoint Measurement. Conjoint measurement (CM) is concerned with combining a set of independent variables in some functional form to predict the values of the dependent variable. CM starts with the consumer's overall or global judgments about a set of product alternatives. It then performs a rather remarkable job of decomposing his/her original evaluations into separate and compatible utility scales by which original global judgments can be reconstituted. Being able to separate overall judgments into psychological components in this manner can provide a manager with valuable information about the relative importance of various attributes of a product. It also permits determination of consumer part-worth utilities for different attributes and their levels. Understanding of such part-worths would enable the manager to decide what the basic features of the product offerings should be, and at what levels should these features be offered.

Green and Wind [17], Green and Srinivasan [16], and Green and Rao [15] discuss applications of conjoint measurement to concept testing and product design, and identify the potential uses of the conjoint measurement approach. They also demonstrate how the conjoint measurement methodology can be integrated with other multidimensional scaling techniques to help managers develop product-positioning stragegies.

Both conjoint measurement and LINMAP are based on the utility principle of compensatory choice behavior, where the attributes of the alternatives are simultaneously combined into one functional form. In the additive form of the conjoint measurement a linear utility function is specified, whereas LINMAP proposes a quadratic utility function. An evaluation of these models in DSS framework is presented in Table 1. Another method, proposed by Zionts [59], assumes a linear utility function even though a true utility function is not estimated. This approach is explicated briefly below.

Zionts' Method for Choosing from Among Discrete Alternatives. This method assumes that the set of available alternatives can be listed and evaluated based on different criteria or objectives. The method assumes that the decision maker wants to maximize an unspecified concave function of insatiable objectives.

The initial step consists of maximizing a weighted sum of objectives and finding a preferred solution (the initial weights may be assigned arbitrarily). This preferred solution becomes the reference solution in the next stage of the decision process. This reference solution is compared to the sequence of alternatives one at a time and the decision maker is asked to choose the preferred solution in each pair. Based on his responses, a new set of weights consistent with the choices made are chosen. This set of weights is then used to obtain a new preferred solution (alternative) which maximizes the corresponding weighted sum of the objectives. This new solution is presented to the decision maker. If this solution is preferred to the reference solution, the new solution becomes the reference solution (alternative). Otherwise a different reference alternative is found. The process continues until no alternative is preferred over the current reference solution. At that point, the most preferred solution and a ranking based on the decision maker's responses is prepared to assist in the decision-making process. The algorithm identifies a small subset of preferred alternatives which should contain the "best" alternative. The algorithm terminates when the number of alternatives 


\section{Integrating Multiple Criteria Decision Making Models}

remaining are very small. The method is not restricted to situations where the underlying preferences are additive.

The above method has been used satisfactorily when the number of alternatives are large. Several substantial improvements in the method have been made [30]. An evaluation of this model in DSS framework is presented in Table 1. Finally, this section will conclude by discussing another important MCDM model, goal programming.

Goal-Programming Models. Goal programming (GP) is an extension of a mathematical programming model. In this method the decision maker attempts to achieve a "satisfactory" level of multiple objectives, rather than the best possible outcome for a single objective (as in linear programming).

The fundamental notion of goal programming involves incorporating all managerial goals into a system model formulation. In the GP formulation, the decision maker specifies acceptable or desirable levels on single attribute values (this is a constraint on one variable), or on combinations of attributes (constraints on more than one variable), and these serve as the primary goals. In GP, instead of attempting to maximize or minimize the objective function directly, as in linear (or nonlinear) programming, the deviations from the desired goals are minimized. General surveys of goal programming are given in Charnes and Cooper [10] and Ignizio [20].

Interactive goal programming, on the other hand, begins with equal weights on all the goals. The weights are then changed to reflect the feedback of the decision maker. For an interactive multiple-objective linear programming model, refer to the Zionts and Wallneius [61] approach. An evaluation of goal programming in DSS framework is presented in Table 1 .

In this section, different methods of dealing with multiattribute or multiple objective problems are explicated. Evidence on the applicability of single MCDM models exists. However, available literature, though limited, indicates that combining these models to solve multiattribute, multicriterion, or multiobjective problems may result in better performance. Some of the applications where more than one model are used are described next.

\section{MCDM Mixed-Modeling Approach}

In their recent work, O'Leary and O'Leary [40] indicated how the conjoint measurement approach can be integrated with goal programming to form a useful decision tool. This algorithmic approach allows the development of models that reflect a manager's decisions and multiple goals. Accordingly, the approach can form the algorithmic core of a DSS. In another study which evaluates the performance of five algorithms for multicriteria decision making, Khairullah and Zionts [29] discuss mixed models. They compared LINMAP, conjoint measurement, ORDREG (ordinal regression), the mixed model (LINMAP and ORDREG), and Ziont's [61] interactive algorithm, on several criteria, including the computational effort required. These models focus on evaluating a set of alternatives to arrive at a preference ranking of either the entire set, or a subset of the available alternatives. They noted that LINMAP, ORDREG, MIXED, and conjoint measurement models performed equally well in predicting true preference order, with the mixed model having a slight edge over the other models. Other researchers working in the marketing field have utilized mixed models to analyze choice decisions [32, 41, 42, 55].

This trend towards mixed modeling can be supported by the recent developments in 


\section{Integrating Multiple Criteria Decision Making Models}

the area of DSS, specifically the development of model management systems. The models (MCDM and other models) used in the DSS are managed as an organizational resource by the model management system. This provides support for model representation and manipulation which refers to the process of automatically selecting, sequencing, and activating the right model units (given a model-bound decision problem) from a model base.

Model manipulation also requires the availability of information concerning the use of the various model units in the model base. The general approach for model management is to store expert knowledge (for selection and sequencing models) in the knowledge base and all related models in the model base. The knowledge base can serve as a front end to the model base supporting the problem exploration and conceptual model building within the general decision problem handling framework. A comprehensive review of model-management techniques can be found in [6]. Thus model-management techniques can be used for combining various MCDM models and can also be used for integrating MCDM models with other models to provide improved solutions to the multicriterion problems. More work in this area is required to use this approach successfully in decision making. The next section describes the role of MCDM models within the framework of DSS.

\section{THE ROLE OF MCDM MODELS IN DSS}

As stated by Zeleny [58, p. 1] the evolving consensus of contributors to MCDM is to "let the human decision maker become a core around which to build our techniques, adapting them to his/her needs and amplifying his/her decision making powers." Letting the man in, via man-machine interface seems to be a process of considerable promise. These objectives of the MCDM models match very closely with the objectives of DSS's. The confluence of these two approaches forms a major resource which decision makers can use in the process of dealing with unstructured problems.

Based on the MCDM models reviewed in the preceding section, important characteristics of these models can be summarized as follows.

1. They permit the analysis of several criteria, where the criteria may be quantifiable (e.g., price), nonquantifiable, (e.g., convenience), or also may be conflicting [24, 49, 59].

2. They allow the decision maker to evaluate criteria and alternatives by such procedures as weighting, ranking, or rating them.

3. They permit the decision maker to whittle down a large set of alternatives to a meaningful size by evaluating the alternatives on various criteria.

4. They can be used to find good or acceptable solutions, compromise solutions, or highconfidence solutions.

5. The modeling techniques become more realistic, more flexible, and more acceptable to decision makers; they permit managers to introduce subjective information during the decision process.

6. MCDM techniques can serve as formal tools for preference surfacing, preference aggregation, negotiation, and mediation, both in friendly and in noncooperative decision situations [23].

Section 2 presented the following reference criteria to assess the effectiveness and performance of the DSS: (i) emphasis on semistructured and unstructured decisions, (ii) should provide control to decision maker, (iii) assistance in all phases of decision making, and (iv) ease of use. To discuss the role of MCDM models in DSS, it is necessary to examine whether or not the MCDM models comply with the above desirable features of DSS. 


\section{Integrating Multiple Criteria Decision Making Models}

MCDM models appear in a variety of structures and forms. Some models that are based on multiobjective programmong techniques are highly structured, while those based on heuristics are applicable to ill-structured problems. However, the inherent capability of MCDM models allows the user to cope with ill-structured problems better than conventional OR/MS models. This notion of "unstructuredness" is further corroborated by Moore and Chang [38] and Bonczek et al. [5]. Thus MCDM models, by their very nature, seem to support the criteria that they are used to solve semistructured and unstructured decision problems.

MCDM models allow consideration of a number of separate objective functions. This implies that usually a multitude of solutions could be recommended for formal analysis by the decision maker. No single mathematically optimum solution can be stipulated. The decision maker is required to play a major role in evaluating, comparing, and ranking of the resulting solutions. The key concepts here are nondenominated solutions, good or acceptable solutions, compromise solutions or high-confidence solutions. Thus the modeling techniques become more realistic, more flexible, and more acceptable to managers. It provides a spectrum of control for the decision maker and allows him/her to introduce subjective information during the solution process. The decision maker becomes an integral part of the loop of solution-process iterations. Thus the model supports an essential characteristic of DSS.

The next question is, do MCDM techniques support all phases of the decisionmaking process? Simon [50] characterizes the decision-making process as having three phases: intelligence, design, and choice. The majority of the DSS's concentrate on the last two phases, decision and choice. Sprague and Carlson [51] point out that little attention has been focused on the design phase. The MCDM models have the potential to deal with this phase. Bui [7], in his recent work, noted that the design issue relies more on the affordability (in terms of time and money) to the user, rather than on the capability of the MCDM to support all phases of the decision-making process. In fact, he pointed out that large-scale MCDM models did cover Simon's three phases.

The next characteristic of DSS to be considered is ease of use. There are at least two factors that contribute to this characteristic: the simplicity of the decision method used, and the ability to interface with the user. Within the domain of MCDM techniques, some are sophisticated while others are easy to use. Sophisticated techniques rely on the knowledge or expertise of the user, who must handle powerful programming techniques. Linear and nonlinear programming models come under this category. In this, the manager has to rely on the expertise around him. The other models, such as the linear additive models, pair-wise comparison methods, and Zionts' interactive approach, are easy to use.

In the last 10 to 15 years the multiple-criteria decision-making models have gained increased acceptance as a useful tool for decision making [19]. Individuals and organizations have increasingly recognized the importance of considering real-world multiple and conflicting objectives. There are numerous successful applications of these models reported in the literature $[19,56]$. Like all other modeling methods, MCDM models have their advantages and disadvantages. When used correctly, they can be a powerful tool for models, solutions, and analysis of real world problems. Some of the difficulties presently limiting the use of these models are the following.

(i) Unavailability and/or the volume of input data required by the model.

(ii) Lack of friendly and powerful user interface required by these models for presenting alternative solutions and collecting preference information from the decision maker. Here it should be pointed out that some interactive MAUT-based models, such as the weighted 
linear additive utility of simple pairwise comparison ratios, are easy to use and learn [7]. However, one might question whether or not these simple models can capture the complexity of the decision problem.

(iii) Relative unfamiliarity and unwillingness on the part of the decision maker to interact directly with the computer system.

The recent surge of interest in decision-support systems and their powerful data bases and user-interface language capabilities may aid in the use of MCDM models. The authors strongly believe that MCDM models come closest to satisfying most of the criteria described in Section 2 for the choice of a model for DSS. Embedding MCDM models in a decision-support system can provide very powerful and useful decision-support capabilities $[21,22,35]$.

\section{CONCLUSIONS AND DIRECTIONS FOR FUTURE RESEARCH}

The article focuses on the multiple-criteria decision-making models and their suitability in the DSS environment. Criteria in selecting the models for DSS are delineated. The study has suggested that the model used should be flexible, and provide feedback and a spectrum of control to the decision maker. The study also points out that MCDM models cannot be considered as stand-alone DSS; rather, MCDM models should be viewed as an integral part of DSS. It particularly advocates that the integration of MCDM-DSS enables a decision maker to devise high-quality solutions to what are often partially formulated or ill-structured problems.

Multiple-criteria decision techniques are very relevant in the situation where the decision maker faces a hard choice among alternatives, in which none of them stands out from the others as clearly the best choice; each alternative is good on some of the criteria but less good on some others. Some of the currently used and recently developed MCDM models in the marketing area are reviewed. The models considered are: the AHP, lexicographic models, the EBA, the MLH model, LINMAP, conjoint measurement, and Ziont's multicriterion approach for choosing discrete alternatives. Each of these models are further evaluated within the DSS framework. Following this, the mixed-modeling approach is discussed. It is pointed out that research on combining more than one MCDM model within the DSS framework is lacking. Existing research, though limited, reveals that the mixed-modeling approach in the DSS environment may perform better than single MCDM models. Finally, the role of the MCDM models in the DSS environment is explicated. It has been pointed out that the MCDM models allow consideration of the decision maker's subjective evaluation, which is often crucial in decision problems. It has been argued that because of their inherent characteristics of "letting the man in" these models can and should play a very important role in building decision-support systems.

\section{APPENDIX:}

\section{Theoretical Basis of the MLH Model}

The MLH model is based on the notion of threshold concept. The concept of individual threshold tolerance, which is fundamental to the MLH model, is defined in the context of individual behavior. The threshold tolerance is assumed to be a rel- 


\section{Integrating Multiple Criteria Decision Making Models}

ative value, related to the attribute value of the alternatives under consideration. The MLH noncompensatory probabilistic model, which generates aggregate estimates of these threshold tolerances, uses sequential processing of ranked attributes, and eliminates the nonchosen alternatives at each stage of the process. MLH is a disaggregate hierarchical model, and is distinct from current lexicographic models in that it does not require the analyst to know a priori individual threshold tolerances (cutoff values).

To understand how the MLH model works, let us clarify some notations. Denote the $i$ th attribute associated with the $r$ th importance ranking of decision maker $n$ as $i(r)$. Furthermore define $A_{i j}^{n}$ as the perceived value (rating) of alternative $j$, with respect to attribute $i(r)$, given by the $n$th individual. Furthermore, associated with any given attribute $i(r)$ there is a critical tolerance $T_{i}^{n}$ between the decision maker $n$ 's evaluation of any alternative on the attribute $i(r)$, and an acceptable standard. This quantity $T_{i}^{n}$ will be considered distributed over the population. These critical tolerances, denoted by $T_{i}$, are parameters of these distributions with certain special properties. Since the model is formulated (see [13]) as a concave programming problem whose solutions are globally optimal, these solutions are precise aggregate estimators of $T_{i}$. If aggregation is to be affected, the information processed by two or more individuals must be compatible. Hence, the standardized individual value is defined as a real number $C_{i j}^{n}$; where $j$ ranges over all alternatives which have not been eliminated, and $i$ ranges over the set of attributes which are arranged in the order of importance. To explain more clearly, at the beginning of the choice process, an individual considers the full set of alternatives denoted by $J(n, 0)$; where $J(n, 0)=\{j \mid j=1,2, \ldots, J\}$; where $n$ denotes the $n$th individual in the sample, and zero indicates that the alternative has been evaluated with respect to no attributes. After an individual implies his cut point (threshold tolerances) for the first ranked attribute to the alternatives, the set of alternatives (which may or may not be reduced) is denoted by $J(n, 1)$. In general, after the application of the first $K$ ranked attributes, the set is $J(n, K)$. Thus the individual standardized values, which are a function of those alternatives still under consideration, are defined as

$$
C_{i j}^{n}=\frac{\max _{m \varepsilon J(n, k)}\left[A_{i m}^{n}\right]-A_{i j}^{n}}{\max _{m \in J(n, k)}\left[A_{i m}^{n}\right]} .
$$

It is clear from the above formation that $C_{i j}^{n}$ lies in the interval $[0,1]$, and that data from two or more individuals is compatible. It may also be noted that once the set $J(n, k)$ is reduced to a single alternative, the values $C_{i j}^{n}$ remain fixed.

Consider now the aspect of the individual's set of threshold tolerances (cut points). Without a loss of generality, these cutpoints may be standardized in the manner of individual values. The standardized individual cut points, which also lie in the interval $[0,1]$, are denoted $\tau_{i}^{n}$ and are called individual tolerances.

The aggregate threshold tolerances, denoted $\tau_{i}$, are central tendency parameters of these distributions $\tau_{i}^{n}$, with certain special properties. MLH generates the estimates of these parameters which are called aggregate tolerances.

Initially, an individual $n$ evaluates the set of alternatives with respect to his first 


\section{Integrating Multiple Criteria Decision Making Models}

ranked attribute $i$. An alternative $j$ will be eliminated if the individual's tolerance (standard cut point) $\tau_{i}^{n}$ is less than the individual's (standardized) value for the alternative, with respect to that attribute. That is, alternative $j$ will be eliminated if

$$
\tau_{i}^{n}<C_{i j}^{n}
$$

and not eliminated if

$$
\tau_{i}^{n} \geq C_{i j}^{n}
$$

Consider now the definition of these sets (2), where the individual tolerances $\tau_{i}^{n}$ are replaced by estimates of the distribution parameters $\tau_{i}$. The application of the parameter estimates to the individual data will simulate the evaluation process for a set of individuals, and the definition of the resulting sets of alternatives remaining. Equations (1) and (2a) indicate that the individual, having assessed the set of available alternatives, determines a set of tolerances associated with each attribute of the alternatives. These criteria for acceptance are based upon his perception of the best available alternatives. This premise is similar to an ideal point model in which the ideal point is a composite of the best available alternatives. The formulation of the MLH model is discussed in [13].

\section{REFERENCES}

[1] Aczel, J. and Saaty, T. L., "Procedures for Synthesizing Ratio Judgements," Journal of Mathematical Psychology, 27, 93-102 (1983).

[2] Barbosa, L. and Hirko, R., "Integration of Algorithmic Aids into Decision Support Systems," MIS-Quarterly, 4, 1-12 (1980).

[3] Benayoun, R. J. de Montgolfier, Tergeny, J., and Larichev, O., "Linear Programming With Multiple Objective Functions: Step Method (STEM)," Mathematical Programming, 1, 366-375 (1971).

[4] Bettman, J. R., "Analyzing Consumer Information Processing Models: A Graph Theoretic Approach," Management Science, 18, 114-128 (1971).

[5] Bonczek, R. H., Holsapple, C. W., and Winston, A. W., "The Evolving Roles of Models," Decision Sciences, 11, 339-356 (1980).

[6] Bu-Hulaiga, M. and Jain, H., "Contemporary Approaches to Model Management in Decision Support Systems," Working Paper, School of Business Administration, University of Wisconsin-Milwaukee, April, 1987.

[7] Bui, T., "Building Effective Multiple Criteria Decision Models: A Decision Support Approach," Systems, Objectives, and Solutions, 4, 3-16 (1984).

[8] Chankong, V. and Haimes, Y. Y., Multiple Objective Decision Making: Theory and Methodology, Elsevier Science Publishing Co., New York, 1983.

[9] Chankong, V., Haimes, Y., Thadathil, J., and Zionts, S., "Multiple Criteria Optimization: A State of the Art Review," in Y. Y. Haimes and V. Chankong, Eds., Decision Making with Multiple Objectives Proceedings, Cleveland, Ohio, Springer-Verlag, New York, 1984, pp. 36-90

[10] Charnes, A. and Cooper L., "Goal Programming and Multiple Objective Optimizations," European Journal of Operation Research, 1 (1977).

[11] Dyer, J. S., and Mulvey, J. M., "Integrating Optimization Models with Information Systems for Decision Support," in J. Bennett, Ed., Building Decision Support Systems, Addison-Wesley, Reading, MA, 1983.

[12] Gensch, D. H. and Javalgi, R. G., "The Influence of Involvement on Disaggregate Attribute Choice Models," Journal of Consumer Research, 14, 71-82 (1987).

[13] Gensch, D. H. and Svestka, J. A., "A Maximum Likelihood Hierarchical Disaggregate Models for Predicting Choices of Individuals," Journal of Mathematical Psychology, 25, $160-178$ (1984). 


\section{Integrating Multiple Criteria Decision Making Models}

[14] Geoffrion, A. M., Dyer, J., and Feinberg, A., "An Interactive Approach for Multicriterion Optimization with an Application to the Operation of an Academic Department," Management Science, 19, 357-368 (1972).

[15] Green, P. and Rao, V., "Conjoint Measurement for Quantifying Judgment Data," Journal of Marketing Research, 8, 355-363 (1971).

[16] Green, P. and Srinivasan, V., "Conjoint Analysis in Consumer Research: Issues and Outlook," Journal of Consumer Research, 5, 103-123 (1978).

[17] Green, P. and Wind, Y., Multiattribute Decisions in Marketing, Dryden Press, Hinsdale, IL, 1973.

[18] Hwang, G. L., Paidy, S. R., Yoon, K., and Masud, A. S. M., "Mathematical Programming with Multiple Objectives: A Tutorial," Computers and Operation Research, 7 (1980).

[19] Ignizio, J. P., "Generalized Goal Programming-An Overview," Computers and Operation Research, 10 (1983).

[20] Ignizio, J. P., Goal Programming and Extensions, Lexington Books, Lexington, MA, 1976.

[21] Jain, H. K., "Role of Multiple Criteria Decision Making Models in Building Decision Support Systems," Proceedings of the Sixteenth Annual Pittsburgh Conference on Modeling and Simulation, 1985, Vol. 16.

[22] Jain, H. K. and Javalgi, R. G., "Models for Management: A DSS Perspective," Proceedings of the National Conference of Decision Sciences Institute, 1986.

[23] Jarke, M., "Knowledge Sharing and Negotiation Support in Multiperson Decision Support Systems," Decision Support Systems, 2, 93-102 (1986).

[24] Jelassi, M., Jarke, M., and Stohr, E., "Designing a Generalized Multiple Criteria Decision Support System," in Y. Y. Haimes and V. Chankong, Eds., Decision Making with Multiple Objectives Proceedings, Cleveland, OH, Springer-Verlag, New York, 1984, pp. 214-235.

[25] Keen, P. G. W., "Decision Support Systems: Translating Analytic Techniques into Useful Tools," Sloan Management Review, 21 (1980).

[26] Keen, P. G. W. and Scott Morton, M. S., Decision Support Systems: An Organizational Perspective, Addison-Wesley, Reading, MA, 1978, pp. 33-59.

[27] Keeney, R. J. and Raiffa, H., Decision Making With Multiple Objectives, Wiley, New York, 1976.

[28] Keeney, R. J., "Decision Analysis: An Overview," Operations Research, 30, 803-838 (1982).

[29] Khairullah, Z. and Zionts, S., "An Experiment with Some Algorithms for Multiple Criteria Decision Making," in G. Fandal and T. Gal, Eds., Multiple Criteria Decision MakingTheory and Applications Proceedings, Hagen/Königswinter, West Germany, SpringerVerlag, New York, 1979, pp. 150-159.

[30] Koksalan, M., Karwan, M. H., and Zionts, S., "Approaches for Discrete Alternative Multiple Criteria Problems for Different Types of Criteria," IIE Transactions (1985).

[31] Landry, M., Daniel P., and Dominique, B., "Can DSS Evolve Without Changing Our View of the Concept of Problem?", Decision Support Systems, 1, 25-36 (1985).

[32] Lussier, D. A. and Olshavsky, R. W., "Task Complexity and Contingent Processing in Brand Choice," Journal of Consumer Research, 6, 154-165 (1979).

[33] MacCrimmon, K. R., "An Overview of Multiple Objective Decision Making," in J. Cochrane and M. Zeleny, Eds., Multiple Criteria Decision Making University of South Carolina Press, Columbia, SC, 1973.

[34] Masud, A. S., and Hwang, C. L., "Interactive Sequential Goal Programming," Journal of the Operations Research Society, 32, 391-400 (1981).

[35] Minch, P. R. and Sanders, L. G., "Computerized Information Systems Supporting Multicriteria Decision Making," Decision Sciences, 17, 395-413 (1986).

[36] Monarchi, D. E., Kisiel, C. C., and Duckstein, L., "Interactive Programming in Water Resources: A Case Study," Water Resources Research, 9, 837-850 (1973).

[37] Monarchi, D. E., Weber, J. E., and Duckstein, L., "An Interactive Multiple Objective Decision Making Aid Using Non-Linear Goal Programming," in M. Zeleny, Ed., Multiple Criteria Decision Making, Operations Research, New York, 1976, p. 123.

[38] Moore, J. H. and Chang, M. G., "Design of Decision Support Systems," Data Base, 12, 40-46 (1980). 


\section{Integrating Multiple Criteria Decision Making Models}

[39] Newell, A. and Simon, H., Human Problem Solving, Prentice-Hall, Englewood Cliffs, NJ, 1972.

[40] O'Leary, D. H., and O'Leary, D. E., "The Use of Conjoint Analysis in the Determination of Goal Programming Weights for a Decision Support System," in Y. Y. Haimes and V. Chankong, Eds., Decision Making with Multiple Objectives Proceedings, Cleveland, $\mathrm{OH}$, Springer-Verlag, New York, 1984, pp. 287-299.

[41] Payne, J. W., "Task Complexity and Contingent Processing in Decision Making: A Replication and Extension," Organizational Behavior and Human Performance, 24, 300-387 (1976).

[42] Russ, F. A., "Consumer Evaluation of Alternative Product Models," unpublished Ph.D. dissertation, Carnegie-Mellon University, 1971.

[43] Saaty, T. L., The Analytic Hierarchy Process, McGraw Hill, New York, 1980.

[44] Saaty, T. L., "The Analytic Hierarchy Process and Health Care Problems," Proceedings of International Conference on Systems Science in Health Care, Montreal, 1980.

[45] Saaty, T. L., Rogers, P. C., and Pell, R., "Portfolio Selection Through Hierarchies," The Journal of Portfolio Management, 6, 16-21 (1979).

[46] Saaty, T. L., "Rank Generation, Preservation and Reversal in the Analytic Hierarchy Decision Process," Decision Sciences, 18, 157-177 (1987).

[47] Saaty, T. L., Decision Making For Leaders, Van Nostrand Reinhold, 1982.

[48] Schroder, H. M., Driver, M. J., and Streufert, S., Human Information Processing, Holt, New York, 1977.

[49] Shakun, M. F., "Formalizing Conflict Resolution in Policy Making," International Journal of General Systems, 7 (1981).

[50] Simon, H., The New Science of Management Decision, Harper and Row Publishers, New York, 1960.

[51] Sprague, R. H. and Carlson, E. D., Building Effective Decision Support Systems, PrenticeHall, Englewood Cliffs, NJ, 1982.

[52] Srinivasan, V. and Schocker, A. D., "Linear Programming Techniques for Multidimensional Analysis of Preferences," Psychometrika, 38, 337-369 (1973).

[53] Tversky, A., "Elimination-by-Aspects: A Theory of Choice," Psychological Review, 79, 281-299 (1972).

[54] Wind, Y. and Saaty, T. L., "Marketing Applications of the Analytic Hierarchy Process," Management Science, 26, 641-658 (1980).

[55] Wright, P. L. and Frederick, B., "Phase Decision Strategies: Sequels to an Initial Screening," in M. K. Starr and M. Zeleny, Eds., Multiple Criteria Decision Making, TIMS-Studies in Management Science, North-Holland, Amsterdam, 1977.

[56] Zeleny, M., Multiple Criteria Decision Making, McGraw-Hill, New York, 1982.

[57] Zeleny, M., "A Selected Bibliography of Works Related to Multiple Criteria Decision Making," in J. L. Cochrane and M. Zeleny, Eds., Multiple Criteria Decision Making, University of South Carolina Press, Columbia, SC, 1973.

[58] Zeleny, M., "Multiple Objectives in Mathematical Programming: Letting the Man In," foreword to special issue of Computers and Operation Research, 7 (1980).

[59] Zionts, S., "A Survey of Multiple Criteria Method for Choosing Among Discrete Alternatives," European Journal of Operation Research, 7, 143-147 (1981).

[60] Zionts, S., "A Report on a Project on Multiple Criteria Decision Making," unpublished.

[61] Zionts, S. and Wallenius, J., "An Interactive Programming Method for Solving the Multiple Criteria Problem," Management Science, 22, 652-663 (1976). 